\title{
Global solar radiation estimation from commonly available meteorological data for Bangladesh
}

\author{
Md. Nazmul Islam Sarkar ${ }^{*}$ (D) and Anwarul Islam Sifat
}

\begin{abstract}
In this study, several regression models were employed to estimate global solar radiation from commonly available meteorological data such as sunshine duration, temperature, precipitation, and cloud cover for 34 meteorological stations of Bangladesh. The models studied were calibrated using five meteorological stations that are providing global solar radiation as well as other meteorological data. Estimated values were also compared with measured values in terms of statistical evaluation indicators like the coefficient of determination $\left(R^{2}\right)$, mean percentage error, mean bias error, root mean square error (RMSE), mean absolute relative error, and $t$ statistic. The statistical analysis showed that the models assessed were well suited to accurately estimate the solar potential. Sunshine duration-based models performed best, and cloud cover-based models performed worst. Among 45 developed models to predict solar radiation, the models with RMSE value lower than 0.2 are recommended for use.
\end{abstract}

Keywords: Global solar radiation, Solar radiation estimation models, Correlation models, Angstrom equation

\section{Background}

Among fossil fuels, Bangladesh has only natural gas that is used in power generation in the country (Ahiduzzaman and Islam 2011). According to Bangladesh Power Development Board (BPDB) (2015), Bangladesh has a total installed capacity of $11,532 \mathrm{MW}$ and among which $62.76 \%$ of the total generation comes from natural gas. Figure 1 shows the total installed power capacity by fuel type for Bangladesh. A large amount of imported furnace oil is burnt to produce $20.45 \%$ of total power which emits a huge amount of $\mathrm{CO}_{2}$ and other toxic gases. The estimated reserve of natural gas which is around 13.75 Tcf would meet the country's demand till 2015. After that there will be a shortage of gas supply which would rise to 4421 MMcfd by 2025 . Therefore, an additional 8.35 Tcf supply of gas is to be met by some other means (Ahiduzzaman and Islam 2011). Geographically, Bangladesh is a low-lying delta and has insignificant amount of hills and high water reservoirs. There is only a single hilly area situated in the southern

*Correspondence: sarkarmni@gmail.com

Institute of Energy, University of Dhaka, Dhaka 1000, Bangladesh part of the country, and the only hydro power plant is situated there (Bangladesh Power Development Board (BPDB) 2015). This plant gives only $230 \mathrm{MW}$ which is only $1.99 \%$ of the total capacity, and there is no other suitable location for anymore large hydro power plants. Fortunately, Bangladesh receives moderate amount of daily sunshine of $4.5-4.8 \mathrm{~kW} \mathrm{~h} / \mathrm{m}^{2}$ which can be proved very significant for a sustainable power sector (Mondal and Islam 2011).

Knowledge of the amount of global solar radiation is essential to utilize solar power. Without prior knowledge of solar radiation, it is impossible to design solar energy systems and models. Local global solar radiation data are also required in many applications, including evapotranspiration estimates, irrigation, and architectural design. In spite of the great importance of solar radiation measurements, due to the high cost, maintenance, and calibration requirements, it is not readily available to the designers of solar energy systems (Almorox and Hontoria 2004). In fact, there is no solar radiation measurement instruments installed in any stations of Bangladesh Meteorological Department. Rather there is Campbell-Stokes sunshine recorder installed in 35 stations of Bangladesh

\section{望 Springer}

(c) 2016 Sarkar and Sifat. This article is distributed under the terms of the Creative Commons Attribution 4.0 International License (http://creativecommons.org/licenses/by/4.0/), which permits unrestricted use, distribution, and reproduction in any medium, provided you give appropriate credit to the original author(s) and the source, provide a link to the Creative Commons license, and indicate if changes were made. 


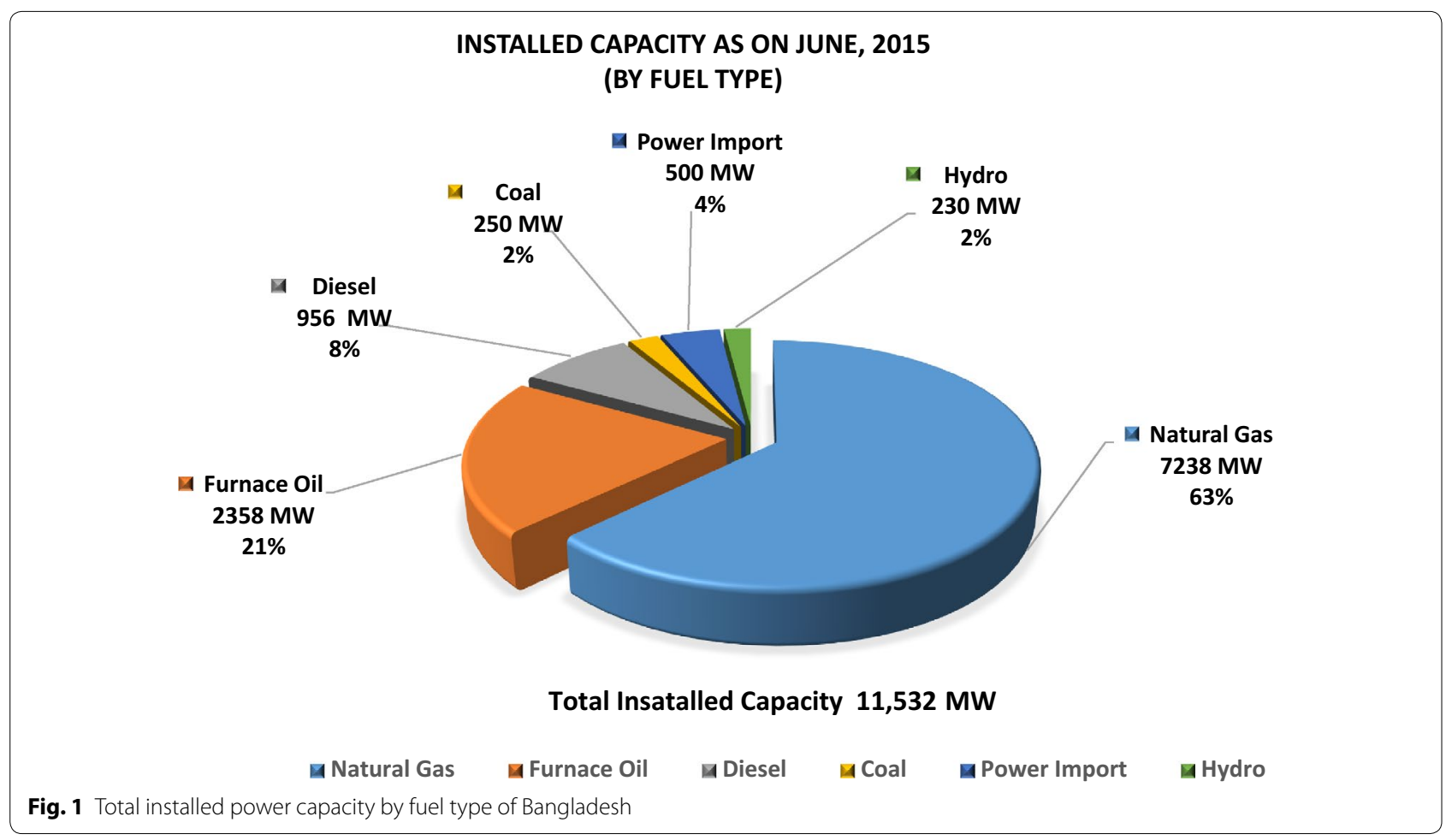

Meteorological Department (BMD) 2015). So there is a need to develop methods for estimating solar radiation from sunshine duration data or from other meteorological parameters like cloud cover, temperature, precipitation, or humidity.

In past, many researchers have studied various meteorological parameters like sunshine hours (Angstrom 1924), air temperature (Hargreaves and Samani 1982), precipitation (DeJong and Stewart 1993), relative humidity (Elagib et al. 1998), and cloudiness (Black 1956) to establish a relationship with solar radiation. Angstrom (1924) gave the first model to estimate solar radiation from sunshine duration data. It was a linear model and used the ratio of average daily global radiation to the corresponding value on a completely clear day. As it was difficult to define a clear day, the model was later modified by Prescott (1940) which replaces clear day radiation with extraterrestrial radiation. This is the well-known Angstom-Prescott (A-P) model widely used by researchers to find solar radiation from sunshine duration ( $\mathrm{Li}$ et al. 2011; Chelbi et al. 2015; Al-Mostafa et al. 2014a). Bakirci (2015) has developed some new empirical models for predicting the monthly mean diffuse solar radiation on a horizontal surface for typical cities in Turkey. Chelbi et al. (2015) have done the solar radiation mapping using sunshine durationbased models and interpolation techniques for Tunisia. Simple correlation for estimating the global solar radiation on horizontal surfaces for India was done by Katiyar and Pandey (2010). Liu et al. (2015) found the changes in the relationship between solar radiation and sunshine duration in large cities of China. Robaa (2008) has done the evaluation of sunshine duration from cloud data in Egypt. Yang et al. (2012) have done the hourly solar irradiance time series forecasting using cloud cover index. Nimnuan and Janjai (2012) have found another approach for estimating average daily global solar radiation from cloud cover in Thailand. New types of simple nonlinear models to compute solar global irradiance from cloud cover amount were found by Badescu and Dumitrescu (2014). Ehnberg and Bollen (2005) have done a simulation of global solar radiation based on cloud observations. Reddy (1974) has developed an empirical method for estimating sunshine from total cloud amount. Morf (2014) has done sunshine and cloud cover predictions based on Markov processes. Babatunde and Aro (1995) have established a relationship between "clearness index" and "cloudiness index" at a tropical station, for instance, Ilorin, Nigeria. AlMostafa et al. (2014b) have done a review of sunshinebased global radiation models. Manzano et al. (2015) established a method to estimate the daily global solar radiation from monthly data. Abraha and Savage (2008) have done a comparison of estimates of daily solar radiation from air temperature range. Temperature-related models were also derived by several researchers such as 
Allen (1997), Liu et al. (2009), Meza and Varas (2000), and Thornton and Running (1999). Both temperatureand precipitation-based models were developed by Bindi and Miglietta (1991), Liu and Scott (2001), Thornton et al. (2000), and Winslow et al. (2001). Unfortunately, there are very few or limited studies on solar radiation estimation models for Bangladesh (Podder et al. 2014; Khan et al. 2014; Khadem et al. 2007; Rahman et al. 1999; Ghosh et al. 2006).

The objective of this study is to derive and validate several expression models for the prediction of monthly averaged daily global radiation on a horizontal surface from commonly available meteorological data and to find the most suitable and adequate model for Bangladesh.

\section{Methodology}

\section{Sunshine duration-based models}

Different regression models to estimate global solar radiation on the horizontal surface from sunshine duration is proposed in the literature (Almorox and Hontoria 2004; Angstrom 1924; Prescott 1940; Akinoglu and Ecevit 1990; Ertekin and Yaldiz 2000; Ampratwum and Dorvlo 1990; Sen 2007). The models we have examined in this work are given in Table 2. We have used the general A-P model and also modified the model for seasonal variations. We did not take higher-degree models greater than third degree as those models fluctuates a lot and do not give proper fit.

In the models in Table $2, H$ is the global solar radiation on horizontal surface $\left(\mathrm{kW} \mathrm{h} / \mathrm{m}^{2}\right), H_{0}$ is the extraterrestrial radiation $\left(\mathrm{kW} \mathrm{h} / \mathrm{m}^{2}\right), S$ is the sunshine duration $(\mathrm{h})$, $S_{0}$ is the day length (h), and $a, b, c$, and $d$ are the empirical coefficients those we have to find.

Extraterrestrial solar radiation $H_{0}$ can be calculated according to Duffie and Beckman (2006):

$$
\begin{aligned}
H_{0}= & \frac{\left(24 \times 3600 G_{\mathrm{sc}}\right)}{\pi}\left(1+0.033 \cos \frac{360 D}{365}\right) \\
& \times\left(\cos \phi \cos \delta \sin \omega_{\mathrm{s}}\right)+\frac{\left(\pi \omega_{\mathrm{s}}\right)}{180} \sin \phi \sin \delta
\end{aligned}
$$

where $G_{\mathrm{sc}}$ is the solar constant $\left(1367 \mathrm{~W} / \mathrm{m}^{2}\right), D$ is the day number of the year counting from first January, $\phi$ is the latitude of the place $\left(^{\circ}\right), \omega_{\mathrm{s}}$ is the sunset hour angle $\left(^{\circ}\right), \delta$ is the solar declination $\left(^{\circ}\right)$.

The value of $\delta$ and $\omega_{\mathrm{S}}$ can be calculated from Eqs. (2) and (3), respectively (Duffie and Beckman 2006):

$$
\begin{aligned}
& \delta=23.45 \sin \left[\frac{360(D+284)}{365}\right] \\
& \omega_{s}=\arccos [-\tan (\delta) \tan (\phi)]
\end{aligned}
$$

The day length $S_{0}$ can be calculated using the value of $\omega_{\mathrm{s}}$ as follows (Duffie and Beckman 2006):

$$
S_{0}=\frac{2}{15} \omega_{\mathrm{s}}
$$

Now, the ratio $H / H_{0}$ can be found by substituting the values of measured global solar radiation $(H)$ and extraterrestrial radiation $H_{0}$. This ratio $H / H_{0}$ is known as clearness index which gives the percentage deflection by the sky of the incoming global radiation and changes in the atmospheric conditions in a given locality (Iqbal 1983).

\section{Sunshine duration and other meteorological parameter-based models}

Solar radiation does not only depend on sunshine duration. It also depends on temperature deviation, precipitation, cloud cover, or extraterrestrial radiation. So, to derive better models for solar radiation estimation, in this study we have proposed few models combining the effects of sunshine duration and other meteorological parameters. Table 5 lists all these models and their coefficients with statistical evaluation.

\section{Cloud cover-based models}

Black (1956) used data of 88 stations from all over the world to develop an empirical relationship between solar radiation and cloud cover. He used the mean monthly values for 88 stations and performed a regression analysis to find the following relationship between $H / H_{0}$ and $C$ :

$$
\frac{H}{H_{0}}=0.803-0.340 C-0.458 C^{2}
$$

Unfortunately, this relationship can produce substantial amount of error as according to Black himself (Black et al. 1954): (1) Mean monthly values were calculated from the maximum numbers of years, and (2) different instruments have been used in different stations, and no attempts have been made to reduce these instruments to a common standard.

Therefore, we have done a regression analysis to establish some new models to estimate solar radiation $(H)$ directly from cloud fraction $(C)$ for Bangladesh. To do this, we have established a relationship between clearness index, $H / H_{0}$, and cloud fraction, $C$. The value of $H / H_{0}$ can be determined similarly as shown in the previous section.

\section{Temperature-based models}

There is a clear relationship between solar radiation and temperature. Higher insolation increases the temperature, and low or no insolation decreases the temperature significantly. Therefore, some relationships can be established between solar radiation and temperature deviation to estimate global solar radiation. Bristow and Campbell (1984) expressed the relationship between clearness 
index $\left(H / H_{0}\right)$ and difference of maximum and minimum air temperatures ( $T_{\max }$ and $T_{\min }$, respectively) $\Delta T$ as:

$$
\frac{H}{H_{0}}=a\left[1-\exp \left(-b \Delta T^{\mathrm{c}}\right)\right]
$$

where $a, b, c$ are empirical coefficients to be found.

Hargreaves and Samani (1982) proposed another relationship between clearness index and temperature difference:

$$
\frac{H}{H_{0}}=a(\sqrt{\Delta T})
$$

Later on, this relationship was modified by various researchers such as Chen et al. (2004) who gave the following relationship:

$$
\frac{H}{H_{0}}=a(\sqrt{\Delta T})+b
$$

Table 7 shows all the empirical models based on temperature to estimate solar radiation. Few new models have also been proposed here in this study.

\section{Temperature- and extraterrestrial radiation-based models}

Extraterrestrial radiation, $H_{0}$, is the amount of solar radiation reaching the atmosphere from the sun. After coming through the atmosphere, it is absorbed by various particles like water vapor and air molecules or reflected from the earth surface. Extraterrestrial radiation is the theoretically possible maximum radiation that would reach the earth surface if the atmosphere was absent (Duffie and Beckman 2006). The temperature difference, $\Delta T$, occurs because of the variation of insolation reaching the earth surface which is directly related with solar radiation, $H$. It can now be assumed that there is a clear and close relationship between solar radiation, extraterrestrial radiation, and temperature difference. Goodin et al. (1999) have proposed the following relationship:

$$
\frac{H}{H_{0}}=a\left[1-\exp \left(-b \frac{\Delta T^{\mathrm{c}}}{H_{0}}\right)\right]
$$

Table 7 shows few empirical models based on temperature and extraterrestrial radiation and their associated statistical evaluation.

\section{Temperature- and precipitation-based models}

Precipitation surely decreases the solar insolation level significantly. Temperature deviation also occurs because of precipitation. DeJong and Stewart (1993) have given the following relationship based on precipitation and temperature difference:

$$
\frac{H}{H_{0}}=a\left(\Delta T^{\mathrm{b}}\right)\left(1+c P+d P^{2}\right)
$$

In this study, we have also proposed few more models based on precipitation and temperature as shown in Table 7 .

\section{Data}

In this work, the measured daily global solar radiation data are taken from Institute of Energy (previously known as Renewable Energy Research Center), University of Dhaka, and Bangladesh Meteorological Department (BMD). Measured time series data of global solar radiation was available for five sites, and sunshine duration, precipitation, cloud cover, and temperature data were available for 34 stations of BMD. Table 1 summarizes the detailed information of the stations and the period of observations of the relevant data. Figure 2 shows the distribution of the stations over the country.

Institute of Energy (RERC) measured global solar radiation from January 2003 to December 2005 using two Eppley PSP pyranometers at 1-min interval for $24 \mathrm{~h}$. Sunshine duration data were recorded using Campbell-Stokes sunshine recorders by both BMD and Institute of Energy. We have used the monthly averaged daily solar radiation data and their corresponding other parameter data, such as sunshine duration, precipitation, and temperature to perform the regression analysis. Only five sites, where measured solar radiation data are available, are taken to find the parameters of the models. Data of all the other sites are used to test the models. DLR satellite time series data (German Aerospace Center 2015) of global solar radiation were also available for ten sites from Solar and Wind Energy Resource Assessment (SWERA) project database (Schillings et al. 2004). NASA's Surface meteorology and Solar Energy (SSE) (NASA 2015) data which are the 22-year average satellite data are also collected. As the DLR data and NASA SSE data are actually estimated data, they were not used to find model parameters, rather they are just used to show a comparison among the results.

\section{Statistical evaluation tools}

In this study, six different statistical quantitative indicators were used to evaluate different models. These quantitative indicators are: the coefficient of determination $\left(R^{2}\right)$, mean percentage error (MPE), mean bias error (MBE), root mean square error (RMSE), mean absolute relative error (MARE), and $t$ statistic $(t$ stat). These indicators can be calculated as follows (Despotovic et al. 2015):

$$
\begin{gathered}
R^{2}=1-\frac{\sum_{i=1}^{n}\left(H_{i, \mathrm{~m}}-H_{i, \mathrm{c}}\right)^{2}}{\sum_{i=1}^{n}\left(H_{i, \mathrm{~m}}-H_{m, \mathrm{avg}}\right)^{2}} \\
\mathrm{MPE}=\frac{1}{n} \sum_{i=1}^{n}\left(\frac{H_{i, \mathrm{c}}-H_{i, \mathrm{~m}}}{H_{i, \mathrm{~m}}} \times 100\right)
\end{gathered}
$$


Table 1 Geographical locations and period of data of the BMD stations

\begin{tabular}{|c|c|c|c|c|c|c|c|c|c|c|}
\hline \multirow[t]{2}{*}{ Name } & \multirow{2}{*}{$\begin{array}{l}\text { WMO } \\
\text { station } \\
\text { no. }\end{array}$} & \multirow{2}{*}{$\begin{array}{l}\text { Latitude } \\
\left({ }^{\circ} \mathrm{N}\right)\end{array}$} & \multirow{2}{*}{$\begin{array}{l}\text { Longitude } \\
\left({ }^{\circ} \mathrm{E}\right)\end{array}$} & \multirow{2}{*}{$\begin{array}{l}\text { Elevation } \\
(\mathrm{m})\end{array}$} & \multicolumn{6}{|c|}{ Period of observation } \\
\hline & & & & & $\begin{array}{l}\text { Measured } \\
\text { radiation }(H)\end{array}$ & $\begin{array}{l}\text { Sunshine } \\
\text { duration }(S)\end{array}$ & $\operatorname{DLR}(H)$ & $\begin{array}{l}\text { Cloud } \\
\text { cover (C) }\end{array}$ & $\begin{array}{l}\text { Tempera- } \\
\text { ture }(T)\end{array}$ & $\begin{array}{l}\text { Precipita- } \\
\text { tion }(P)\end{array}$ \\
\hline Barisal & 41950 & 22.7 & 90.36 & 4 & - & 1967-2013 & $\begin{array}{l}2000 \\
2002- \\
2003\end{array}$ & $\begin{array}{l}1949- \\
2013\end{array}$ & 1949-2013 & 1949-2013 \\
\hline Bhola & 41951 & 22.7 & 90.66 & 5 & - & $1981-2013$ & - & $\begin{array}{l}1966- \\
2013\end{array}$ & 1966-2013 & 1966-2013 \\
\hline Bogra & 41883 & 24.88 & 89.36 & 20 & 2005 & $1961-2013$ & $\begin{array}{l}2000 \\
2002- \\
2003\end{array}$ & $\begin{array}{l}1948- \\
2013\end{array}$ & 1948-2013 & 1950-2013 \\
\hline Chandpur & 41941 & 23.26 & 90.67 & 7 & - & 1977-2013 & - & $\begin{array}{l}1964- \\
2013\end{array}$ & 1964-2013 & 1966-2013 \\
\hline Chittagong & 41978 & 22.34 & 91.79 & 6 & - & $1961-2013$ & $\begin{array}{l}2000 \\
2002- \\
2003\end{array}$ & $\begin{array}{l}1949- \\
2013\end{array}$ & 1949-2013 & 1949-2013 \\
\hline Chuadanga & 41926 & 23.65 & 88.82 & 0 & 2005 & 2003-2013 & - & $\begin{array}{l}2001- \\
2013\end{array}$ & $1989-2013$ & 2003-2013 \\
\hline Comilla & 41933 & 23.48 & 91.19 & 10 & - & $1981-2013$ & - & $\begin{array}{l}1948- \\
2013\end{array}$ & 1948-2013 & 1948-2013 \\
\hline Cox's Bazar & 41992 & 21.46 & 91.98 & 4 & - & $1961-2013$ & $\begin{array}{l}2000 \\
2002- \\
2003\end{array}$ & $\begin{array}{l}1948- \\
2013\end{array}$ & 1948-2013 & $1948-2013$ \\
\hline Dhaka & 41923 & 23.78 & 90.39 & 9 & 2003-2005 & $1961-2013$ & $\begin{array}{l}2000 \\
2002- \\
2003\end{array}$ & $\begin{array}{l}1953- \\
2013\end{array}$ & $1953-2013$ & 1953-2013 \\
\hline Dinajpur & 41863 & 25.63 & 88.66 & 37 & - & 1989-2013 & - & $\begin{array}{l}1948- \\
2013\end{array}$ & 1948-2013 & 1948-2013 \\
\hline Faridpur & 41929 & 23.61 & 89.84 & 9 & - & 1985-2013 & - & $\begin{array}{l}1948- \\
2013\end{array}$ & 1948-2013 & 1948-2013 \\
\hline Feni & 41943 & 23.01 & 91.37 & 8 & - & 1985-2013 & - & $\begin{array}{l}1973- \\
2013\end{array}$ & 1973-2013 & 1973-2013 \\
\hline Hatiya & 41963 & 22.29 & 91.13 & 4 & - & $1985-2013$ & - & $\begin{array}{l}1966- \\
2013\end{array}$ & 1966-2013 & $1966-2013$ \\
\hline Ishurdi & 41907 & 24.12 & 89.04 & 14 & - & 1985-2013 & - & $\begin{array}{l}1961- \\
2013\end{array}$ & $1961-2013$ & 1962-2013 \\
\hline Jessore & 41936 & 23.17 & 89.22 & 7 & - & $1967-2013$ & - & $\begin{array}{l}1948- \\
2013\end{array}$ & 1948-2013 & 1948-2013 \\
\hline Khepupara & 41984 & 21.98 & 90.22 & 3 & - & 1988-2013 & - & $\begin{array}{l}1974- \\
2013\end{array}$ & 1975-2013 & 1975-2013 \\
\hline Khulna & 41947 & 22.8 & 89.58 & 4 & - & 1984-2013 & - & $\begin{array}{l}1948- \\
2013\end{array}$ & 1948-2013 & 1948-2013 \\
\hline Kutubdia & 41989 & 21.83 & 91.84 & 6 & - & 1984-2013 & - & $\begin{array}{l}1985- \\
2013\end{array}$ & 1985-2013 & 1977-2013 \\
\hline Madaripur & 41939 & 23.17 & 90.18 & 5 & - & 1988-2013 & - & $\begin{array}{l}1977- \\
2013\end{array}$ & 1977-2013 & 1977-2013 \\
\hline $\begin{array}{l}\text { Maijdee } \\
\text { Court }\end{array}$ & 41953 & 22.83 & 91.08 & 6 & - & 1985-2013 & - & $\begin{array}{l}1951- \\
2013\end{array}$ & $1951-2013$ & 1951-2013 \\
\hline Mongla & 41958 & 22.43 & 89.66 & 4 & - & $2001-2013$ & - & $\begin{array}{l}1991- \\
2013\end{array}$ & 1989-2013 & 1991-2013 \\
\hline Mymensingh & 41886 & 24.75 & 90.41 & 19 & - & 1979-2013 & - & $\begin{array}{l}1948- \\
2013\end{array}$ & 1948-2013 & 1948-2013 \\
\hline Patuakhali & 41960 & 22.36 & 90.34 & 3 & - & 1985-2013 & - & $\begin{array}{l}1973- \\
2013\end{array}$ & 1973-2013 & 1975-2013 \\
\hline Rajshahi & 41895 & 24.35 & 88.56 & 20 & - & 1979-2013 & $\begin{array}{l}2000 \\
2002- \\
2003\end{array}$ & $\begin{array}{l}1964- \\
2013\end{array}$ & 1964-2013 & 1964-2013 \\
\hline
\end{tabular}


Table 1 continued

\begin{tabular}{|c|c|c|c|c|c|c|c|c|c|c|}
\hline \multirow[t]{2}{*}{ Name } & \multirow{2}{*}{$\begin{array}{l}\text { WMO } \\
\text { station } \\
\text { no. }\end{array}$} & \multirow{2}{*}{$\begin{array}{l}\text { Latitude } \\
\left({ }^{\circ} \mathrm{N}\right)\end{array}$} & \multirow{2}{*}{$\begin{array}{l}\text { Longitude } \\
\left({ }^{\circ} \mathrm{E}\right)\end{array}$} & \multirow{2}{*}{$\begin{array}{l}\text { Elevation } \\
\text { (m) }\end{array}$} & \multicolumn{6}{|c|}{ Period of observation } \\
\hline & & & & & $\begin{array}{l}\text { Measured } \\
\text { radiation }(H)\end{array}$ & $\begin{array}{l}\text { Sunshine } \\
\text { duration }(S)\end{array}$ & $\operatorname{DLR}(H)$ & $\begin{array}{l}\text { Cloud } \\
\text { cover }(C)\end{array}$ & $\begin{array}{l}\text { Tempera- } \\
\text { ture }(T)\end{array}$ & $\begin{array}{l}\text { Precipita- } \\
\text { tion }(P)\end{array}$ \\
\hline Rangamati & 41966 & 22.67 & 92.2 & 63 & 2005 & 1987-2013 & $\begin{array}{l}2000 \\
2002- \\
2003\end{array}$ & $\begin{array}{l}1957- \\
2013\end{array}$ & $1957-2013$ & $1957-2013$ \\
\hline Rangpur & 41859 & 25.72 & 89.26 & 34 & - & 1979-2013 & $\begin{array}{l}2000 \\
2002- \\
2003\end{array}$ & $\begin{array}{l}1954- \\
2013\end{array}$ & $1957-2013$ & 1954-2013 \\
\hline Sandwip & 41964 & 22.5 & 91.46 & 6 & - & $1987-2013$ & - & $\begin{array}{l}1967- \\
2013\end{array}$ & $1966-2013$ & 1966-2013 \\
\hline Satkhira & 41946 & 22.68 & 89.07 & 6 & 2005 & 1984-2013 & $\begin{array}{l}2000 \\
2002- \\
2003\end{array}$ & $\begin{array}{l}1948- \\
2013\end{array}$ & $1948-2013$ & 1948-2013 \\
\hline Sitakunda & 41965 & 22.64 & 91.64 & 4 & - & $1977-2013$ & - & $\begin{array}{l}1977- \\
2013\end{array}$ & $1977-2013$ & 1977-2013 \\
\hline Srimangal & 41915 & 24.29 & 91.73 & 23 & - & 1986-2013 & - & $\begin{array}{l}1948- \\
2013\end{array}$ & $1948-2013$ & 1949-2013 \\
\hline Syedpur & 41858 & 25.75 & 88.92 & 0 & - & 2003-2013 & - & $\begin{array}{l}1991- \\
2013\end{array}$ & $1991-2013$ & 2003-2013 \\
\hline Sylhet & 41891 & 24.88 & 91.93 & 35 & - & $1962-2013$ & $\begin{array}{l}2000 \\
2002- \\
2003\end{array}$ & $\begin{array}{l}1956- \\
2013\end{array}$ & $1956-2013$ & 1956-2013 \\
\hline Tangail & 41909 & 24.15 & 89.55 & 10 & - & 1987-2013 & - & $\begin{array}{l}1987- \\
2013\end{array}$ & $1987-2013$ & 1987-2013 \\
\hline Teknaf & 41998 & 20.87 & 92.26 & 4 & - & 1977-2013 & - & $\begin{array}{l}1977- \\
2013\end{array}$ & $1977-2013$ & 1977-2013 \\
\hline
\end{tabular}

$$
\begin{aligned}
& \mathrm{MBE}=\frac{1}{n} \sum_{i=1}^{n}\left(H_{i, \mathrm{~m}}-H_{i, \mathrm{c}}\right) \\
& \mathrm{RMSE}=\sqrt{\frac{1}{n} \sum_{i=1}^{n}\left(H_{i, \mathrm{~m}}-H_{i, \mathrm{c}}\right)^{2}} \\
& \text { MARE }=\frac{1}{n} \sum_{i=1}^{n}\left|\frac{H_{i, \mathrm{~m}}-H_{i, \mathrm{c}}}{H_{i, \mathrm{~m}}}\right| \\
& t \text { stat }=\sqrt{\frac{(n-1) \mathrm{MBE}^{2}}{\mathrm{RMSE}^{2}-\mathrm{MBE}^{2}}}
\end{aligned}
$$

where $H_{i, \mathrm{~m}}$ and $H_{i, \mathrm{c}}$ are the $i$ th measured and calculated values, respectively $\left(\mathrm{kW} \mathrm{h} / \mathrm{m}^{2}\right), H_{\mathrm{m} \text {,avg }}$ is the average of the calculated and measured values $\left(\mathrm{kW} \mathrm{h} / \mathrm{m}^{2}\right)$, and $n$ is the number of observations. For better data modeling, MPE, MBE, MARE, $t$ stat, and RMSE should be closer to zero, but $R^{2}$ should approach 1.0 as closely as possible.

\section{Results and discussion}

The empirical models for solar radiation estimation are given in Tables 2 and 7. The regression equations developed and their associated statistical evaluation are given in Tables 3, 4, 5, and 6 and in Tables 8, 9, and 10. In Table 3, sunshine duration-based annual models are shown whereas seasonal regression results for summer (February-September) and winter (October-January) are shown in Table 4. Comparing all the results of these models, we can see that sunshine duration-based seasonal models give much better performance than annual models. Among annual models quadratic model (model \# 2 of Table 3) gave the best fit, whereas among seasonal models the third-degree model (model \# 9 of Table 4) was the best. In the both cases, the linear models also performed well and were pretty close to the best model. Logarithmic, exponential, and power models were also good, but their RMSE was comparatively high than the linear or quadratic models. Table 5 shows combined sunshine durationbased and other meteorological parameter-based models and their statistical evaluation. Among these models, model no. 18 gave the best fit. Model nos. 13, 14, and 17 were also pretty good with very low RMSE value. 


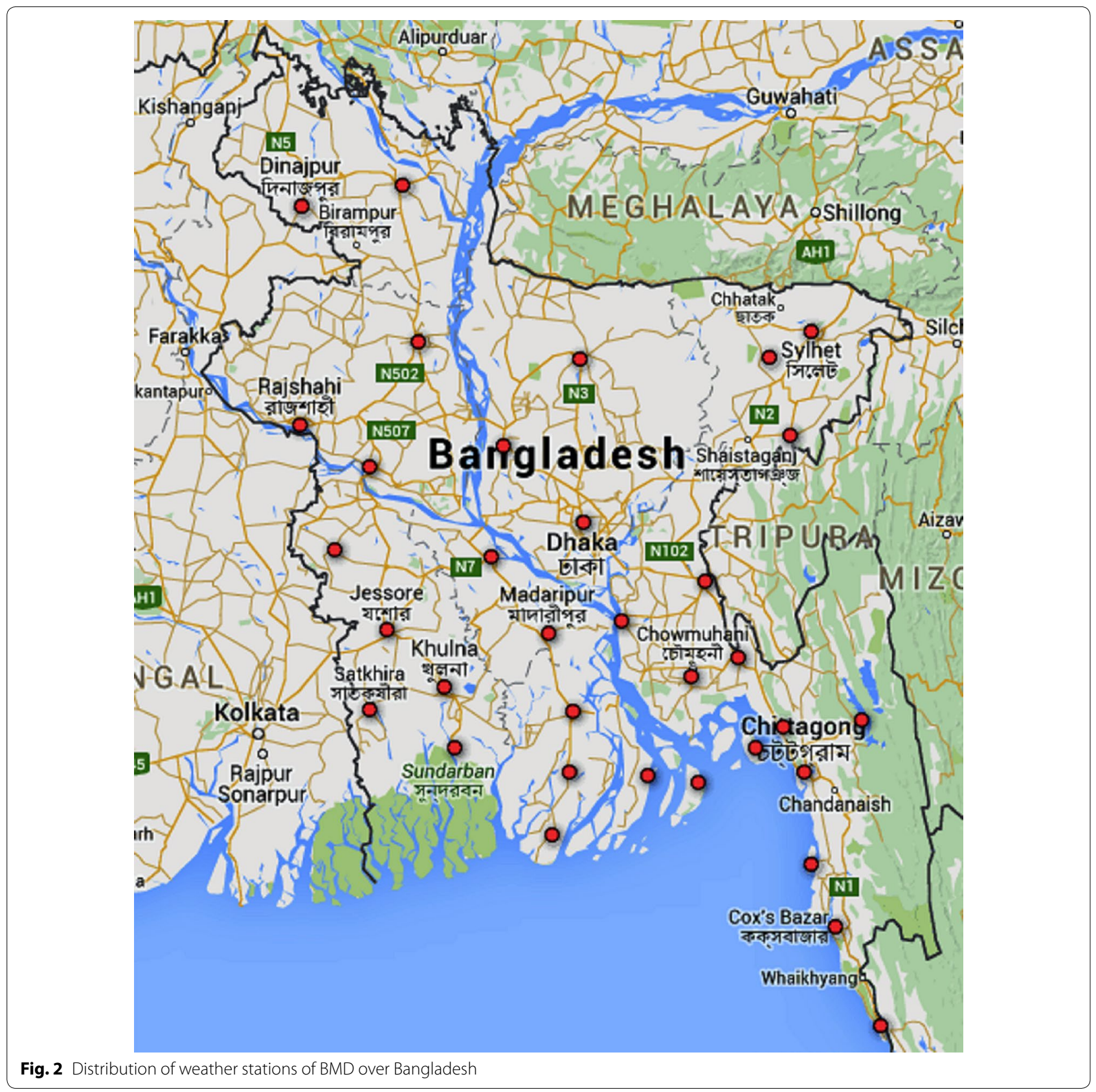

The cloud cover-based models are given in Table 6. Linear, quadratic, third-, fourth-, and fifth-degree models along with logarithmic, exponential, and power models are evaluated. We can see that the higher-degree models perform better than linear or quadratic models. Table 8 shows the temperature-based models and their statistical evaluation. We can see that model nos. 31, 33, 36, and 39 perform best. Temperature- and extraterrestrial radiation-based models and temperature-with-precipitationbased models are given in Tables 9 and 10. Very good fits are achieved with model nos. 41, 43, and 45 .
Figure 3 illustrates the RMSE values of all 45 models. We can see that the sunshine duration-based seasonal models have the lowest RMSE values. All the models having RMSE values lower than 0.2 will perform pretty well. The cloud cover models (model no. 22-29) did not perform well having a higher range of RMSE values from 0.25 to 0.45 .

The estimation of solar radiation depends on the data available for a particular site. According to our results for different data sets, we propose the following equations for estimation of global solar radiation for Bangladesh: 
Table 2 Empirical models to calculate solar radiation from sunshine duration

\begin{tabular}{lll}
\hline Models & Regression equations & Source \\
\hline Linear & $H / H_{0}=a+b\left(S / S_{0}\right)$ & Angstrom (1924), Prescott (1940) \\
Quadratic & $H / H_{0}=a+b\left(S / S_{0}\right)+c\left(S / S_{0}\right)^{2}$ & Akinoglu and Ecevit (1990) \\
Third-degree & $H / H_{0}=a+b\left(S / S_{0}\right)+c\left(S / S_{0}\right)^{2}+d\left(S / S_{0}\right)^{3}$ & Ertekin and Yaldiz (2000) \\
Logarithmic & $H / H_{0}=a+b \times \log \left(S / S_{0}\right)$ & Ampratwum and Dorvlo (1990) \\
Exponential & $H / H_{0}=a \times \exp \left[b\left(S / S_{0}\right)\right]$ & Almorox and Hontoria (2004) \\
Power & $H / H_{0}=a\left(S / S_{0}\right)^{b}$ & Sen (2007) \\
\hline
\end{tabular}

Table 3 Sunshine duration-based annual regression results and their statistical evaluation

\begin{tabular}{|c|c|c|c|c|c|c|c|c|}
\hline Model no. & Model type & Equations & $R^{2}$ & MBE & MPE & RMSE & MARE & $t$ stat \\
\hline 1 & Linear & $H / H_{0}=0.4256\left(S / S_{0}\right)+0.2199$ & 0.8888 & 0.0183 & 0.3446 & 0.2404 & 0.3446 & 0.2538 \\
\hline 2 & Quad. & $H / H_{0}=0.749\left(S / S_{0}\right)^{2}-0.3752\left(S / S_{0}\right)+0.417$ & 0.8920 & 0.0216 & 0.3276 & 0.2369 & 0.0440 & 0.3049 \\
\hline 3 & Cubic & $H / H_{0}=3.1987\left(S / S_{0}\right)^{3}-4.4223\left(S / S_{0}\right)^{2}+2.3158\left(S / S_{0}\right)-0.0306$ & 0.8899 & 0.0231 & 0.3246 & 0.2393 & 0.0471 & 0.3226 \\
\hline 4 & Expo. & $H / H_{0}=0.2673 \exp \left[0.9422\left(S / S_{0}\right)\right]$ & 0.8913 & 0.0261 & 0.1707 & 0.2376 & 0.0494 & 0.3673 \\
\hline 5 & Log. & $H / H_{0}=0.2152 \ln \left(S / S_{0}\right)+0.5916$ & 0.8729 & 0.0153 & 0.3850 & 0.2570 & 0.0560 & 0.1981 \\
\hline 6 & Power & $H / H_{0}=0.6093\left(S / S_{0}\right)^{0.478}$ & 0.8804 & 0.0233 & 0.1998 & 0.2493 & 0.0537 & 0.3125 \\
\hline
\end{tabular}

Table 4 Sunshine duration-based seasonal regression models and their statistical evaluation

\begin{tabular}{|c|c|c|c|c|c|c|c|c|c|}
\hline $\begin{array}{l}\text { Model } \\
\text { no. }\end{array}$ & Model type & Seasons & Equations & $R^{2}$ & MBE & MPE & RMSE & MARE & $t$ stat \\
\hline \multirow[t]{2}{*}{7} & \multirow[t]{2}{*}{ Linear } & $\begin{array}{l}\text { Summer (February- } \\
\text { September) }\end{array}$ & $H / H_{0}=0.5077\left(S / S_{0}\right)+0.1915$ & \multirow[t]{2}{*}{0.96833} & \multirow[t]{2}{*}{0.00131} & \multirow[t]{2}{*}{0.09172} & \multirow[t]{2}{*}{0.12833} & \multirow[t]{2}{*}{0.02795} & \multirow[t]{2}{*}{0.03394} \\
\hline & & $\begin{array}{l}\text { Winter (October- } \\
\text { January) }\end{array}$ & $H / H_{0}=0.8805\left(S / S_{0}\right)-0.1174$ & & & & & & \\
\hline \multirow[t]{2}{*}{8} & \multirow[t]{2}{*}{ Quadratic } & $\begin{array}{l}\text { Summer (February- } \\
\text { September) }\end{array}$ & $H / H_{0}=0.5469\left(S / S_{0}\right)^{2}-0.0692\left(S / S_{0}\right)+0.3315$ & \multirow[t]{2}{*}{0.97693} & \multirow[t]{2}{*}{0.0023} & \multirow[t]{2}{*}{0.05783} & \multirow[t]{2}{*}{0.10953} & \multirow[t]{2}{*}{0.02242} & \multirow[t]{2}{*}{0.06984} \\
\hline & & $\begin{array}{l}\text { Winter (October- } \\
\text { January) }\end{array}$ & $H / H_{0}=9.3987\left(S / S_{0}\right)^{2}-11.518\left(S / S_{0}\right)+3.9476$ & & & & & & \\
\hline \multirow[t]{2}{*}{9} & \multirow[t]{2}{*}{$\begin{array}{l}\text { Third } \\
\text { degree }\end{array}$} & $\begin{array}{l}\text { Summer (February- } \\
\text { September) }\end{array}$ & $\begin{array}{l}H / H_{0}=-1.6819\left(S / S_{0}\right)^{3}+3.2167\left(S / S_{0}\right)^{2} \\
-1.4324\left(S / S_{0}\right)+0.5545\end{array}$ & \multirow[t]{2}{*}{0.98538} & \multirow[t]{2}{*}{-0.00178} & \multirow[t]{2}{*}{0.09116} & \multirow[t]{2}{*}{0.08719} & \multirow[t]{2}{*}{0.01302} & \multirow[t]{2}{*}{0.06798} \\
\hline & & $\begin{array}{l}\text { Winter (October- } \\
\text { January) }\end{array}$ & $\begin{array}{l}H / H_{0}=1290\left(S / S_{0}\right)^{3}-2585.5\left(S / S_{0}\right)^{2} \\
+1723.8\left(S / S_{0}\right)-381.77\end{array}$ & & & & & & \\
\hline \multirow[t]{2}{*}{10} & \multirow[t]{2}{*}{ Exponential } & $\begin{array}{l}\text { Summer (February- } \\
\text { September) }\end{array}$ & $H / H_{0}=0.2523 \exp \left[1.1088\left(S / S_{0}\right)\right]$ & \multirow[t]{2}{*}{0.97284} & \multirow[t]{2}{*}{0.00425} & \multirow[t]{2}{*}{0.04336} & \multirow[t]{2}{*}{0.11886} & \multirow[t]{2}{*}{0.02535} & \multirow[t]{2}{*}{0.11858} \\
\hline & & $\begin{array}{l}\text { Winter (October- } \\
\text { January) }\end{array}$ & $H / H_{0}=0.1341 \exp \left[1.8733\left(S / S_{0}\right)\right]$ & & & & & & \\
\hline \multirow[t]{2}{*}{11} & \multirow[t]{2}{*}{ Logarithmic } & $\begin{array}{l}\text { Summer (February- } \\
\text { September) }\end{array}$ & $H / H_{0}=0.254 \ln \left(S / S_{0}\right)+0.6323$ & \multirow[t]{2}{*}{0.95165} & \multirow[t]{2}{*}{-0.00049} & \multirow[t]{2}{*}{0.1278} & \multirow[t]{2}{*}{0.1586} & 0.03468 & 0.01023 \\
\hline & & $\begin{array}{l}\text { Winter (October- } \\
\text { January) }\end{array}$ & $H / H_{0}=0.5763 \ln \left(S / S_{0}\right)+0.705$ & & & & & & \\
\hline 12 & Power & $\begin{array}{l}\text { Summer (February- } \\
\text { September) }\end{array}$ & $H / H_{0}=0.6617\left(S / S_{0}\right)^{0.5567}$ & 0.96336 & 0.00293 & 0.05992 & 0.13805 & 0.029 & 0.07044 \\
\hline & & $\begin{array}{l}\text { Winter (October- } \\
\text { January) }\end{array}$ & $H / H_{0}=0.7715\left(S / S_{0}\right)^{1.227}$ & & & & & & \\
\hline
\end{tabular}


Table 5 Combined sunshine duration and other meteorological parameter-based proposed models and their coefficients with error test values

\begin{tabular}{|c|c|c|c|c|c|c|c|c|c|c|}
\hline Model no. & Equations & $a$ & $b$ & $c$ & $R^{2}$ & MPE & MBE & RMSE & MARE & $t$ stat \\
\hline 13 & $H / H_{0}=a\left(S / S_{0}\right)^{b}+c C$ & 1.339 & 3.267 & 0.4219 & 0.9146 & 0.066105 & 0.006986 & 0.171177 & 0.037596 & 0.135465 \\
\hline 14 & $H / H_{0}=a\left(S / S_{0}\right)^{2}+b C^{2}$ & 1.006 & 0.4028 & - & 0.9023 & 0.295246 & 0.006515 & 0.188949 & 0.038023 & 0.114425 \\
\hline 15 & $H / H_{0}=a\left(S / S_{0}\right)^{5}+b P^{c}$ & 1.41 & 0.1989 & 0.1082 & 0.8997 & 0.215373 & 0.00887 & 0.197046 & 0.039099 & 0.149449 \\
\hline 16 & $H / H_{0}=a\left(S / S_{0}\right)^{3}+P / b$ & 1.4113 & 1.131 & - & 0.8976 & -0.00125 & 0.007672 & 0.189384 & 0.04183 & 0.134476 \\
\hline 17 & $H / H_{0}=a\left(S / S_{0}\right)^{b}+c H_{0}$ & 0.9802 & 3.555 & 0.03149 & 0.906 & -0.27016 & 0.000564 & 0.191613 & 0.035362 & 0.009765 \\
\hline 18 & $H / H_{0}=a\left(S / S_{0}\right)^{b}+c \sqrt{H_{0}}$ & 0.8906 & 3.988 & 0.1102 & 0.9352 & 0.195275 & 0.000947 & 0.154964 & 0.032697 & 0.020278 \\
\hline 19 & $H / H_{0}=a\left(S / S_{0}\right)^{b}+c \Delta T^{2}$ & 0.3834 & 0.162 & 0.001501 & 0.9063 & 0.15129 & 0.012653 & 0.152788 & 0.030127 & 0.275613 \\
\hline 20 & $H / H_{0}=a\left(S / S_{0}\right)+b \sqrt{\Delta T}$ & 0.1133 & 0.1377 & - & 0.8994 & 0.174281 & 0.01076 & 0.159612 & 0.033007 & 0.224097 \\
\hline 21 & $H / H_{0}=a \sqrt{\left(S / S_{0}\right)}+a \sqrt{\Delta T}$ & 0.1274 & - & - & 0.8872 & 0.607408 & -0.00757 & 0.179579 & 0.037655 & 0.139899 \\
\hline
\end{tabular}

Table 6 Cloud cover-based models and their error estimations

\begin{tabular}{|c|c|c|c|c|c|c|c|c|}
\hline $\begin{array}{l}\text { Model } \\
\text { no. }\end{array}$ & Model type & Equations & $R^{2}$ & MBE & MPE & MARE & RMSE & $t$ stat \\
\hline 22 & Linear & $H / H_{0}=-0.2302 C+0.5713$ & 0.993695408 & 0.021340195 & 0.656623 & 0.071691 & 0.33156 & 0.213912 \\
\hline 23 & Quadratic & $H / H_{0}=-0.4585 C^{2}+0.2087 C+0.4942$ & 0.995392681 & 0.015789962 & 0.490597 & 0.058503 & 0.283438 & 0.185052 \\
\hline 24 & Third degree & $H / H_{0}=1.9104 C^{3}-3.1785 C^{2}+1.3352 C+0.3669$ & 0.99540268 & 0.01358356 & 0.448265 & 0.048137 & 0.28313 & 0.159303 \\
\hline 25 & Fourth degree & $\begin{array}{l}H / H_{0}=-11.449 C^{4}+23.22 C^{3} \\
-16.729 C^{2}+4.7227 C+0.0963\end{array}$ & 0.995752521 & 0.000808352 & 0.800828 & 0.054337 & 0.272144 & 0.009851 \\
\hline 26 & Fifth degree & $\begin{array}{l}H / H_{0}=120.19 C^{5}-299.15 C^{4}+281.59 C^{3} \\
-124.07 C^{2}+25.073 C-1.3032\end{array}$ & 0.997452146 & 0.015181073 & 0.113112 & 0.034455 & 0.210776 & 0.239501 \\
\hline 27 & Logarithmic & $H / H_{0}=-0.083 \ln (C)+0.386$ & 0.990582324 & 0.016444457 & 0.887773 & 0.090946 & 0.405233 & 0.1347 \\
\hline 28 & Exponential & $H / H_{0}=0.5819 \exp (-0.509 C)$ & 0.993189811 & 0.036221558 & 0.347545 & 0.072874 & 0.344598 & 0.350561 \\
\hline 29 & Power & $H / H_{0}=0.3862 C^{-0.184}$ & 0.989958374 & 0.037624897 & 0.44366 & 0.091626 & 0.418442 & 0.299433 \\
\hline
\end{tabular}

\section{Sunshine duration-based models:} Annual model:

$$
H / H_{0}=0.4256\left(S / S_{0}\right)+0.2199
$$

Seasonal model:

For summer (February-September):

$$
H / H_{0}=0.5077\left(S / S_{0}\right)+0.1915
$$

For winter (October-January):

$$
H / H_{0}=0.8805\left(S / S_{0}\right)-0.1174
$$

Cloud cover-based model: The fifth-degree model performed best, but considering the simplicity we can recommend third-degree model for solar radiation estimation:

$$
H / H_{0}=1.9104 C^{3}-3.1785 C^{2}+1.3352 C+0.3669
$$

Temperature-based model: For temperature-based model, Bristow and Campbell type model can be used:

$$
H / H_{0}=0.2525 \cdot \exp (0.07019 \Delta T)
$$

If all the meteorological data are available for a site, then any model with a low RMSE value can be chosen for solar radiation estimation. Figure 3 illustrates the comparative RMSE values of all the models.

For an assumption of the performance of the models, we have estimated solar radiation for Bogra station and plotted the results in Fig. 4. Measured solar radiation for Bogra is available only for a year though, the figure clearly shows that the sample models perform quite well. From Fig. 5, we can see that the NASA SSE and DLR data have a higher RMSE values and did not perform well compared to the estimated model values. Therefore, it is recommended not to use NASA SSE data or DLR satellite data in renewable energy feasibility study directly. If any of the meteorological data set is available, then it is better to use models with low RMSE studied here in this work to get a better estimation of solar radiation. 
Table 7 Temperature-, extraterrestrial radiation-, and precipitation-based empirical models and their parameters

\begin{tabular}{|c|c|c|}
\hline Equations & Parameter & Reference \\
\hline \multicolumn{3}{|l|}{ Temperature-based models } \\
\hline$H / H_{0}=a\left[1-\exp \left(-b\left(\Delta T^{c}\right)\right)\right]$ & $a, b, c$ & Bristow and Campbell (1984) \\
\hline$H / H_{0}=a \times \exp (b \Delta T)$ & $a, b$ & Proposed model \\
\hline$H / H_{0}=a\left[1-\exp \left(-b\left(\Delta T^{\mathrm{a}}\right)\right)\right]$ & $a, b$ & Proposed model \\
\hline$H / H_{0}=a \times \exp (b \Delta T)+c \times \exp (d \Delta T)$ & $a, b, c, d$ & Proposed model \\
\hline$H / H_{0}=a(\sqrt{\Delta T})$ & $a$ & Hargreaves and Samani (1982) \\
\hline$H / H_{0}=a(\sqrt{\Delta T})+b$ & $a, b$ & Chen et al. (2004) \\
\hline$H / H_{0}=a \Delta T^{b}$ & $a, b$ & Proposed model \\
\hline$H / H_{0}=a \Delta T^{b}+c$ & $a, b, c$ & Proposed model \\
\hline$H / H_{0}=a \Delta T^{2}+b \Delta T+c$ & $a, b, c$ & Proposed model \\
\hline$H / H_{0}=a \Delta T^{3}+b \Delta T^{2}+c \Delta T+d$ & $a, b, c, d$ & Proposed model \\
\hline \multicolumn{3}{|c|}{ Temperature-and extraterrestrial radiation-based models } \\
\hline$H / H_{0}=a\left[1-\exp \left(-b\left(\Delta T^{c}\right) / H_{0}\right)\right]$ & $a, b, c$ & Goodin et al. \\
\hline$H / H_{0}=a(\sqrt{\Delta T})\left(1+b H_{0}+c\left(H_{0}\right)^{2}\right)$ & $a, b, c$ & Proposed model \\
\hline$H / H_{0}=a+b \Delta T+c H_{0}$ & $a, b, c$ & Proposed model \\
\hline Temperature-and precipitation-based models & & Proposed model \\
\hline$H / H_{0}=a\left(\Delta T^{b}\right)\left(1+c P+d P^{2}\right)$ & $a, b, c, d$ & DeJong and Stewart (1993) \\
\hline$H / H_{0}=a+b \Delta T+c P$ & $a, b, c$ & Proposed model \\
\hline$H / H_{0}=a\left(\Delta T^{b}\right)+c\left(P^{d}\right)$ & $a, b, c, d$ & Proposed model \\
\hline
\end{tabular}

Table 8 Temperature-based models and their coefficients with error test values

\begin{tabular}{|c|c|c|c|c|c|c|c|c|c|c|c|}
\hline $\begin{array}{l}\text { Model } \\
\text { no. }\end{array}$ & Equations & $a$ & $b$ & $c$ & $d$ & $R^{2}$ & MPE & MBE & RMSE & MARE & $t$ stat \\
\hline 30 & $H / H_{0}=a\left[1-\exp \left(-b(\Delta T)^{c}\right)\right]$ & 6.842 & 0.02002 & 0.59 & - & 0.8637 & 0.317626 & -0.00023 & 0.171864 & 0.038084 & 0.004467 \\
\hline 31 & $H / H_{0}=a \times \exp (b \Delta T)$ & 0.2525 & 0.07019 & - & - & 0.8961 & 0.197066 & 0.006198 & 0.167799 & 0.036362 & 0.122597 \\
\hline 32 & $H / H_{0}=a\left[1-\exp \left(-b(\Delta T)^{a}\right)\right]$ & 0.8347 & 0.1412 & - & - & 0.8811 & 1.477691 & -0.04888 & 0.185846 & 0.037303 & 0.904051 \\
\hline 33 & $H / H_{0}=a \times \exp (b \Delta T)$ & 0.2531 & 0.06996 & -1.907 & -1.381 & 0.8961 & 0.192121 & 0.006394 & 0.167813 & 0.036402 & 0.12647 \\
\hline 34 & $\begin{array}{l}+c \times \exp (d \Delta T) \\
H / H_{0}=a\left(\Delta T^{0.5}\right)\end{array}$ & 0.1596 & - & - & - & 0.8784 & 0.131653 & 0.008627 & 0.193591 & 0.038634 & 0.147948 \\
\hline 35 & $H / H_{0}=a\left(\Delta T^{0.5}\right)+b$ & 0.1761 & -0.04091 & - & - & 0.8845 & 1.44731 & -0.0472 & 0.181962 & 0.036747 & 0.89087 \\
\hline 36 & $H / H_{0}=a \Delta T^{b}$ & 0.1381 & 0.569 & - & - & 0.8919 & 0.196359 & 0.00489 & 0.171956 & 0.038205 & 0.094353 \\
\hline 37 & $H / H_{0}=a \Delta T^{b}+c$ & 0.004524 & 1.679 & 0.2933 & - & 0.8959 & 0.197305 & 0.00627 & 0.167929 & 0.036417 & 0.123925 \\
\hline 38 & $H / H_{0}=a \Delta T^{2}+b \Delta T+c$ & 0.001263 & 0.01089 & 0.2741 & - & 0.896 & 0.194758 & 0.00637 & 0.167952 & 0.036419 & 0.12589 \\
\hline 39 & $H / H_{0}=a \Delta T^{3}+b \Delta T^{2}+c \Delta T+d$ & 0.0009056 & -0.02235 & 0.2108 & -0.2714 & 0.8984 & 0.263633 & 0.003942 & 0.169964 & 0.036145 & 0.076945 \\
\hline
\end{tabular}

Table 9 Temperature- and extraterrestrial radiation-based models and their coefficients and error estimation

\begin{tabular}{|c|c|c|c|c|c|c|c|c|c|c|}
\hline Model no. & Equations & $a$ & $b$ & $c$ & $R^{2}$ & MPE & MBE & RMSE & MARE & $t$ stat \\
\hline 40 & $H / H_{0}=a\left[1-\exp \left(-b\left(\Delta T^{c} / H_{0}\right)\right)\right]$ & 0.5268 & 0.6639 & 1.688 & 0.8204 & 0.223397 & 0.017537 & 0.23263 & 0.053098 & 0.250746 \\
\hline 41 & $H / H_{0}=a(\sqrt{\Delta T})\left(1+b H_{0}+c\left(H_{0}\right)^{2}\right)$ & -0.006515 & -5.961 & 0.3323 & 0.93 & 0.49945 & -0.00891 & 0.152122 & 0.033675 & 0.194633 \\
\hline 42 & $H / H_{0}=a+b \Delta T+c H_{0}$ & 0.1534 & 0.0334 & 40.002771 & 0.8975 & 0.191296 & $6.24 \mathrm{E}-05$ & 50.171798 & 0.039023 & 0.001204 \\
\hline
\end{tabular}


Table 10 Temperature-with-precipitation-based models, their coefficients, and error test values

\begin{tabular}{|c|c|c|c|c|c|c|c|c|c|c|c|}
\hline $\begin{array}{l}\text { Model } \\
\text { no. }\end{array}$ & Equations & $a$ & $b$ & c & $d$ & $R^{2}$ & MPE & MBE & RMSE & MARE & $t$ stat \\
\hline 43 & $H / H_{0}=a\left(\Delta T^{b}\right)\left(1+c P+d\left(P^{2}\right)\right)$ & 0.1388 & 0.5576 & 0.000655 & $-1.9 \mathrm{E}-06$ & 0.9121 & 0.09509 & 0.004932 & 0.155113 & 0.031219 & 0.105515 \\
\hline 44 & $H / H_{0}=a+b \Delta T+c P$ & 0.1976 & 0.03139 & -0.000007117 & - & 0.8944 & 0.19521 & 0.006017 & 0.168413 & 0.036981 & 0.118561 \\
\hline 45 & $H / H_{0}=a\left(\Delta T^{\mathrm{b}}\right)+c\left(P^{d}\right)$ & 0.001275 & 2.244 & 0.2371 & 0.04886 & 0.9224 & 0.138848 & -0.00579 & 0.156364 & 0.033018 & 0.122823 \\
\hline
\end{tabular}

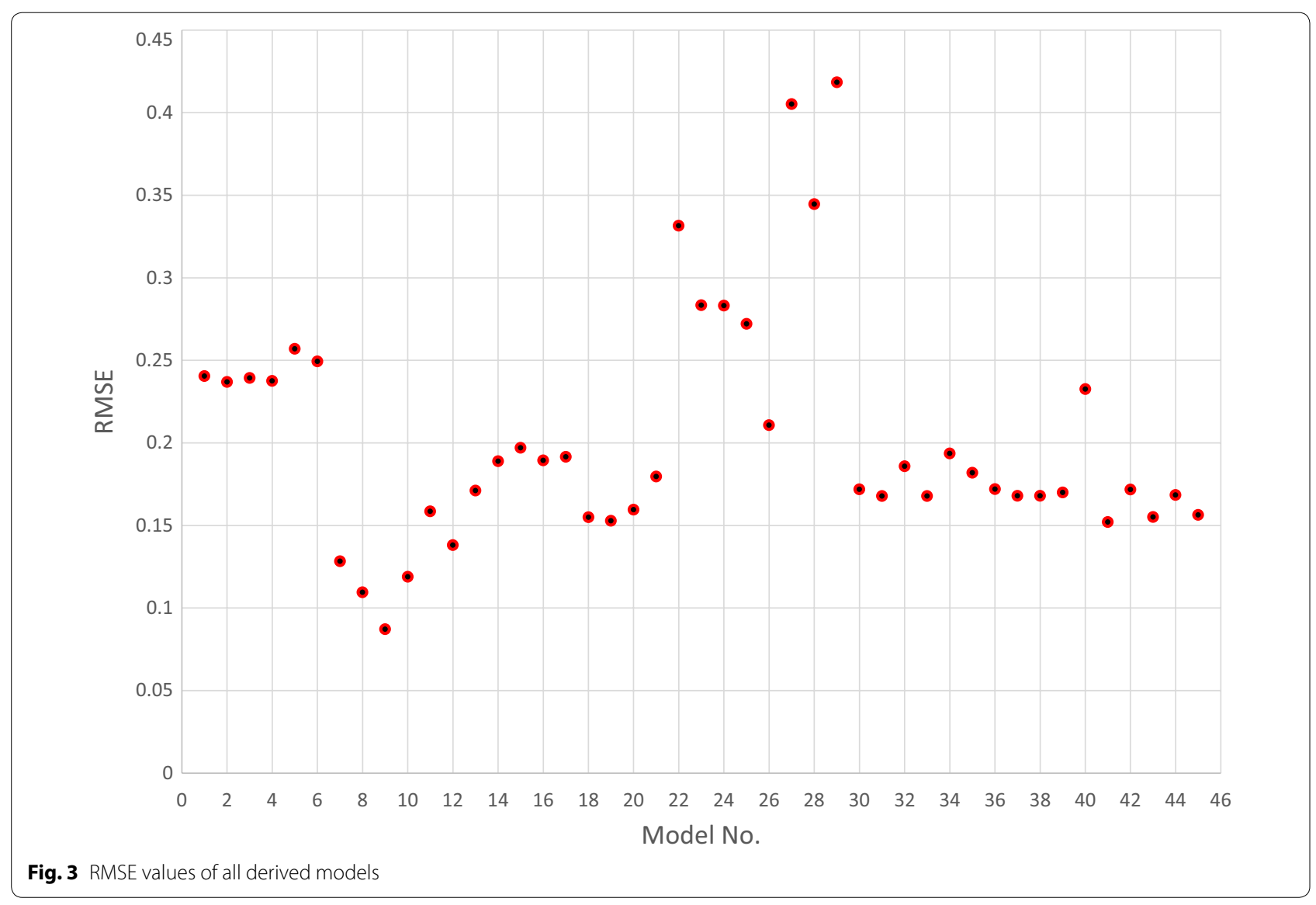




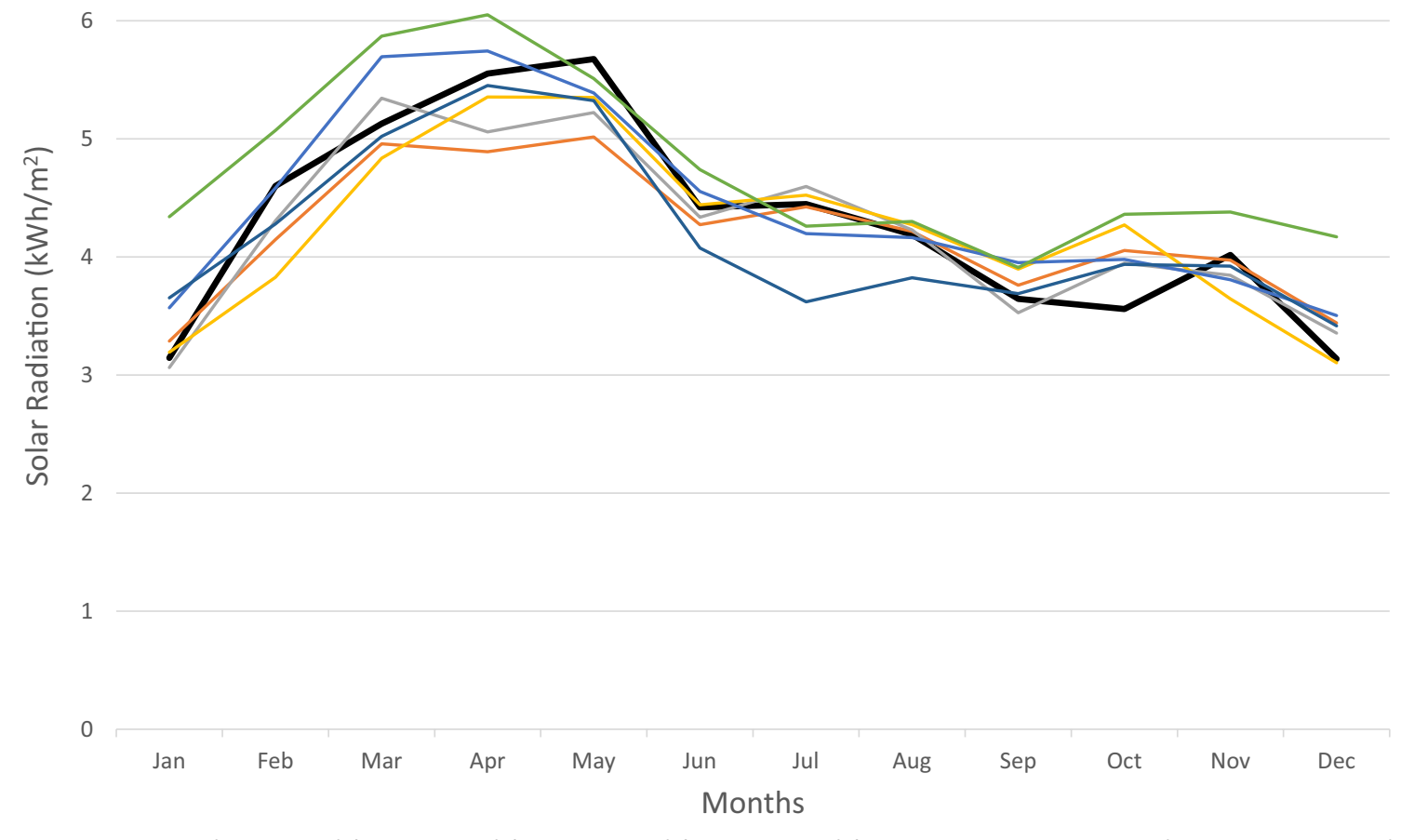

Fig. 4 Comparison of different model performance and satellite data with measured values for Bogra, Bangladesh

\section{7}

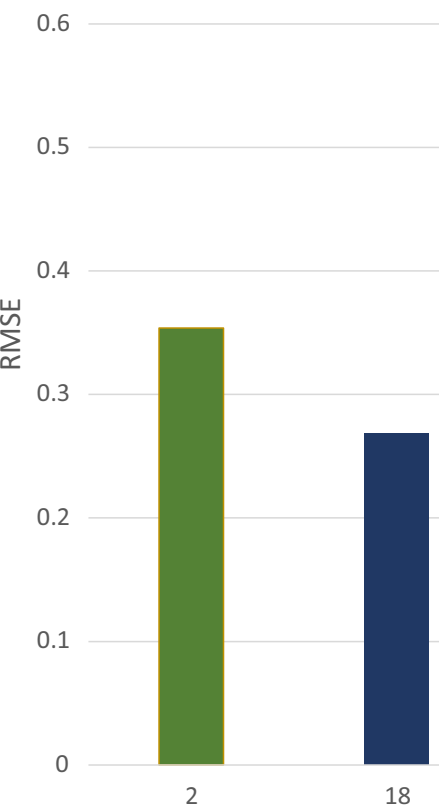

18

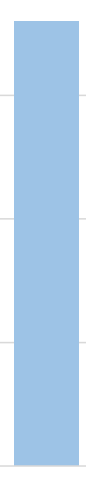

24

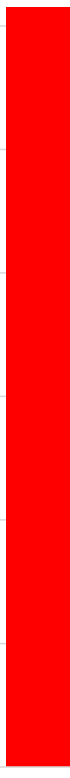

NASA

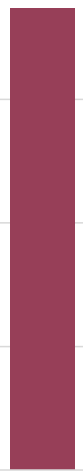

DLR

Models

Fig. 5 RMSE values of different models and satellite data with measured values for Bogra, Bangladesh 


\section{Conclusions}

Bangladesh is a small developing country with very less investment in technology to measure solar radiation all over the country. Therefore, estimating solar radiation from commonly available meteorological data is of great significance. In this work, we have developed 45 models to predict solar radiation from commonly available meteorological data. The estimation of solar radiation depends on the data available for a particular site. According to our results for different data sets, we recommend the use of Eqs. (17)-(21) if only a single data set is available. If there are more data sets, then other models can be chosen. Models with RMSE values lower than 0.2 can be used to predict solar radiation in Bangladesh.

\section{Acknowlegements}

We would like to thank Institute of Energy, University of Dhaka, Bangladesh Meteorological Department, and Bangladesh Agricultural Research Council for their data and support.

\section{Competing interests}

Both authors declare that they have no competing interests.

Received: 23 November 2015 Accepted: 10 February 2016 Published online: 25 February 2016

\section{References}

Abraha, M. G., \& Savage, M. J. (2008). Comparison of estimates of daily solar radiation from air temperature range for application in crop simulations. Agricultural and Forest Meteorology, 148(3), 401-416. doi:10.1016/j. agrformet.2007.10.001.

Ahiduzzaman, M., \& Islam, A. S. (2011). Greenhouse gas emission and renewable energy sources for sustainable development in Bangladesh. Renewable and Sustainable Energy Reviews, 15, 4659-4666.

Akinoglu, B., \& Ecevit, A. (1990). Construction of a quadratic model using modified Ångstrom coefficients to estimate global solar radiation. Solar Energy, $45,85-92$.

Allen, R. G. (1997). Self-calibrating method for estimating solar radiation from air temperature. Journal of Hydrologic Engineering, 2(2), 56-67. doi:10.1061/(ASCE)1084-0699(1997)2:2(56).

Almorox, J., \& Hontoria, C. (2004). Global solar radiation estimation using sunshine duration in Spain. Energy Conversion and Management, 45, 1529-1535.

Al-Mostafa, Z., Maghrabi, A., \& Al-Shehri, S. (2014a). Sunshine-based global radiation models: A review and case study. Energy Conversion and Management, 84, 209-216.

Al-Mostafa, Z. A., Maghrabi, A. H., \& Al-Shehri, S. M. (2014b). Sunshine-based global radiation models: A review and case study. Energy Conversion and Management, 84, 209-216. doi:10.1016/j.enconman.2014.04.021.

Ampratwum, D., \& Dorvlo, A. (1990). Estimation of solar radiation from the number of sunshine hours. Applied Energy, 62, 161-167.

Angstrom, A. (1924). Solar and terrestrial radiation. Quarterly Journal of Royal Meteorological Society, 50, 121-125.

Babatunde, E. B., \& Aro, T. O. (1995). Relationship between "clearness index" and "cloudiness index" at a tropical station (llorin, Nigeria). Renewable Energy, 6(7), 801-805. doi:10.1016/0960-1481(94)00087-M.

Badescu, V., \& Dumitrescu, A. (2014). New types of simple non-linear models to compute solar global irradiance from cloud cover amount. Journal of Atmospheric and Solar-Terrestrial Physics, 117, 54-70. doi:10.1016/j. jastp.2014.05.010.

Bakirci, K. (2015). Models for the estimation of diffuse solar radiation for typical cities in Turkey. Energy, 82, 827-838. doi:10.1016/j.energy.2015.01.093.
Bangladesh Meteorological Department, Agargaon, Dhaka, Bangladesh. (2015). http://www.bmd.gov.bd/.

Bangladesh Power Development Board (BPDB). (2015). http://www.bpdb.gov. $\mathrm{bd} /$ key statistics.htm.

Bindi, M., \& Miglietta, F. (1991). estimating daily global radiation from air temperature and rainfall measurement. Climate Research, 1, 117-124.

Black, J. (1956). The distribution of solar radiation over the earth's surface. Archiv für Meteorologie, Geophysik und Bioklimatologie, Serie B, 7, 165-189.

Black, J., Bonython, C., \& Prescott, J. (1954). Solar radiation and the duration of sunshine. Quarterly Journal of the Royal Meteorological Society, 80, 231-235.

Bristow, K. L., \& Campbell, G. S. (1984). On the relationship between incoming solar radiation and daily maximum and minimum temperature. Agricultural and Forest Meteorology, 31(2), 159-166. doi:10.1016/0168-1923(84)90017-0.

Chelbi, M., Gagnon, Y., \& Waewsak, J. (2015). Solar radiation mapping using sunshine duration-based models and interpolation techniques: Application to Tunisia. Energy Conversion and Management, 101, 203-215.

Chen, R., Ersi, K., Yang, J., Lu, S., \& Zhao, W. (2004). Validation of five global radiation models with measured daily data in China. Energy Conversion and Management, 45(11-12), 1759-1769. doi:10.1016/j. enconman.2003.09.019.

DeJong, R., \& Stewart, D. (1993). Estimating global radiation from common meteorological variables in Western Canada. Canadian Journal of Plant Science, 73, 509-518.

Despotovic, M., Nedic, V., Despotovic, D., \& Cvetanovic, S. (2015). review and statistical analysis of different global solar radiation sunshine models. Renewable and Sustainable Energy Reviews, 52, 1869-1880.

Duffie, J., \& Beckman, W. (2006). Solar engineering of thermal processes (3rd ed.) Hoboken: Wiley.

Ehnberg, J. S. G., \& Bollen, M. H. J. (2005). Simulation of global solar radiation based on cloud observations. Solar Energy, 78(2), 157-162. doi:10.1016/j. solener.2004.08.016.

Elagib, N., Babiker, S., \& Alvi, S. (1998). New empirical models for global solar radiation over Bahrain. Energy Conversation and Management, 39(8), 827-835.

Ertekin, C., \& Yaldiz, O. (2000). Comparison of some existing models for estimating global solar radiation for Antalya (Turkey). Energy Conversation and Managementt, 41, 311-330.

German Aerospace Center (DLR) data. (2015). http://www.dlr.de/tt/desktopdefault.aspx/tabid-2885/4422_read-6548/.

Ghosh, H. R., Mariam, L., Sadia, M., Khadem, S. K., Bhowmik, N. C., \& Hussain, M. (2006). Estimation of monthly averaged daily and hourly global \& diffuse radiation for Bangladesh. Dhaka University Journal of Science, 54(1), 109-113.

Goodin, D. G., Hutchinson, J. M. S., Vanderlip, R. L., \& Knapp, M. C. (1999). Estimating solar irradiance for crop modeling using daily air temperature data. Agronomy Journal, 91(5), 845-851. doi:10.2134/agronj1999.915845x.

Hargreaves, G. H., \& Samani, Z. A. (1982). Estimating potential evapotranspiration. Journal of the Irrigation and Drainage Division, 108(3), 225-230.

Iqbal, M. (1983). An introduction to solar radiation. London: Academic Press.

Katiyar, A. K., \& Pandey, C. K. (2010). Simple correlation for estimating the global solar radiation on horizontal surfaces in India. Energy, 35(12), 5043-5048. doi:10.1016/j.energy.2010.08.014.

Khadem, S., Ullah, S., Aditya, S., Ghosh, H., \& Mariam, L. (2007). Country report of solar and wind energy resource assessment (SWERA) Bangladesh. Dhaka: Renewable Energy Research Center, University of Dhaka.

Khan, M. A., Huque, S., \& Mohammad, A. (2014). A neural network model for estimating global solar radiation on horizontal surface. In International conference on electrical information and communication technology (EICT), pp. 1-4. doi:10.1109/EICT.2014.6777857.

Li, H., Maa, W., Lian, Y., Wang, X., \& Zhao, L. (2011). Global solar radiation estimation with sunshine duration in Tibet, China. Renewable Energy, 36, 3141-3145.

Liu, J., Linderholm, H., Chen, D., Zhou, X., Flerchinger, G. N., Yu, Q., et al. (2015). Changes in the relationship between solar radiation and sunshine duration in large cities of China. Energy, 82, 589-600. doi:10.1016/j. energy.2015.01.068.

Liu, X., Mei, X., Li, Y., Wang, Q., Jensen, J. R., Zhang, Y., et al. (2009). Evaluation of temperature-based global solar radiation models in China. 
Agricultural and Forest Meteorology, 149(9), 1433-1446. doi:10.1016/j. agrformet.2009.03.012.

Liu, D. L., \& Scott, B. J. (2001). Estimation of solar radiation in Australia from rainfall and temperature observations. Agricultural and Forest Meteorology, 106(1), 41-59. doi:10.1016/50168-1923(00)00173-8.

Manzano, A., Martín, M. L., Valero, F., \& Armenta, C. (2015). A single method to estimate the daily global solar radiation from monthly data. Atmospheric Research, 166, 70-82. doi:10.1016/j.atmosres.2015.06.017.

Meza, F., \& Varas, E. (2000). Estimation of mean monthly solar global radiation as a function of temperature. Agricultural and Forest Meteorology, 100(2-3), 231-241. doi:10.1016/50168-1923(99)00090-8.

Mondal, M., \& Islam, A. S. (2011). Potential and viability of grid-connected solar PV system in Bangladesh. Renewable Energy, 36, 1869-1874.

Morf, H. (2014). Sunshine and cloud cover prediction based on Markov processes. Solar Energy, 110, 615-626. doi:10.1016/j.solener.2014.09.044.

NASA. (2015). Surface meteorology and solar energy data. https://eosweb.larc. nasa.gov/sse/.

Nimnuan, P., \& Janjai, S. (2012). An approach for estimating average daily global solar radiation from cloud cover in Thailand. Procedia Engineering, 32, 399-406. doi:10.1016/j.proeng.2012.01.1285. ISEEC.

Podder, S., Khan, R. S., Mohon, S. M. A. A., Hussain, M. J., \& Basher, E. (2014). Solar radiation approximation using sunshine hour at patenga, Bangladesh. In International conference on electrical and computer engineering (ICECE), pp. 321-324. doi:10.1109/ICECE.2014.7026996.

Prescott, J. (1940). Evaporation from a water surface in relation to solar radiation. Transactions of the Royal Society of South Australia, 64, 114-125.

Rahman, M. M., Uddin, M. A., \& Islam, M. W. (1999). Solar beam radiation estimate's correlation for Bangladesh. Indian Journal of Radio \& Space Physics, $28,277-285$.
Reddy, S. J. (1974). An empirical method for estimating sunshine from total cloud amount. Solar Energy, 15(4), 281-285. doi:10.1016/0038-092X(74)90017-6.

Robaa, S. M. (2008). Evaluation of sunshine duration from cloud data in Egypt. Energy, 33(5), 785-795. doi:10.1016/j.energy.2007.12.001.

Schillings, C., Meyer, R., \& Trieb, F. (2004). Final report of solar and wind energy resource assessment (SWERA). United Nations Environment Programme (UNEP)

Sen, Z. (2007). Simple nonlinear solar irradiation estimation model. Renewable Energy, 32, 342-350.

Thornton, P. E., Hasenauer, H., \& White, M. A. (2000). Simultaneous estimation of daily solar radiation and humidity from observed temperature and precipitation: An application over complex terrain in Austria. Agricultural and Forest Meteorology, 104(4), 255-271. doi:10.1016/S0168-1923(00)00170-2.

Thornton, P. E., \& Running, S. W. (1999). An improved algorithm for estimating incident daily solar radiation from measurements of temperature, humidity, and precipitation. Agricultural and Forest Meteorology, 93(4), 211-228. doi:10.1016/S0168-1923(98)00126-9.

Winslow, J. C, Jr., Hunt, E. R., \& Piper, S. C. (2001). A globally applicable model of daily solar irradiance estimated from air temperature and precipitation data. Ecological Modelling, 143(3), 227-243. doi:10.1016/ S0304-3800(01)00341-6.

Yang, D., Jirutitijaroen, P., \& Walsh, W. M. (2012). Hourly solar irradiance time series forecasting using cloud cover index. Solar Energy, 86(12), 3531-3543. doi:10.1016/j.solener.2012.07.029. Solar Resources.

\section{Submit your manuscript to a SpringerOpen ${ }^{\odot}$ journal and benefit from:}

- Convenient online submission

- Rigorous peer review

- Immediate publication on acceptance

- Open access: articles freely available online

- High visibility within the field

- Retaining the copyright to your article

Submit your next manuscript at $\boldsymbol{\wedge}$ springeropen.com 